Die Entwicklung einer chinesischen Corporate Social Responsibility

\title{
Arbeiten in China - Spielen in Deutschland
}

\author{
Erste chinesische Vorreiterunternehmen beginnen sich mit \\ Corporate Social Responsibility (CSR) zu beschäftigen. Und \\ auch die politische Führung entdeckt CSR als Steuerungsele- \\ ment eines verträglicheren Wirtschaftens. Sowohl regulatori- \\ sche als auch freiwillige Initiativen kennzeichnen die Ansätze \\ eines eigenständigen CSR-Verständnisses. \\ Von Ingo Schoenheit und Axel Nordmann
}

\begin{abstract}
A rbeiten in China - Spielen in Deutschland lautete der Titel einer Veranstaltung des Hamburger Museums der Arbeit, auf der am Thema Spielzeug exemplarisch die aktuellen Verwerfungen internationaler Produktions- und Konsumstrukturen diskutiert wurden. Über 50 Prozent des in Deutschland gekauften Spielzeugs werden inzwischen in China unter Bedingungen produziert, die in vielen Fällen als menschenunwürdig angesehen werden. Neben der Spielwarenindustrie lassen insbesondere die Textilund Sportartikelindustrie, aber auch die Unterhaltungselektronik- und Computerindustrie in China fertigen.

Insbesondere wenn renommierte Weltmarken in China produzieren lassen, sehen sie sich dem Verdacht ausgesetzt, dass die international geltenden sozialen Mindeststandards unterlaufen werden. „No Sweatshops“ und „No Logo“ sind die inzwischen etablierten Schlüsselbegriffe der Anti Corporate Movements. Ihr Protest richtet sich in der Regel nicht gegen China oder die dort verantwortlichen Manager und Politiker, sondern gegen herausgehobene global agierende Unternehmen, denen auch die Verantwortung für die sozialen und ökologischen Bedingungen bei ihren Zulieferfirmen zugeschrieben wird (Schoenheit 2007).
\end{abstract}

\section{CSR-Initiativen in China}

Vor diesem Hintergrund ist China innerhalb der europäischen CSR-Diskussion zu einer Metapher geworden. Es steht für billige und zugleich zunehmend qualifizierte Arbeitskräfte, die immer mehr Unternehmen und ganze Industriezweige dazu verleiten, vergleichsweise teure Arbeitsplätze insbesondere in Europa abzubauen und - so das Bild - nach China zu verlagern. Aber auch 1,6 Milliarden potenzielle Kunden locken Unternehmen, die in Europa und in den USA gegen das Phänomen der Marktsättigung ankämpfen, nach China. Beide Standortvorteile, großer Markt und niedriges Lohnniveau, sind die entscheidenden Gründe dafür, dass China weltweit zum größten Empfängerland von Direktinvestitionen geworden ist.

Für europäische und speziell auch deutsche Unternehmen zeichnet sich in Zusammenhang mit ihrem China-Engagement ein schwieriger Spagat ab. Auf der einen Seite wollen sie von den Kostenvorteilen des Standorts China profitieren, die sich aufgrund der niedrigen Lohn-, Sozial- und Umweltstandards ergeben. Auf der anderen Seite stehen sie unter dem Druck einer kritischen öffentlichen Beobachtung und müssen die Reputation der Marken des Unternehmens schützen (Schoenheit 2006).

Während zurzeit nur noch in kleinen Zirkeln darüber nachgedacht wird, ob es angesichts offensichtlicher Demokratiedefizite und Menschenrechtsverletzungen zu verantworten sei, überhaupt in China zu produzieren oder durch den Export von Waren den Konsum in China anzukurbeln, setzen nicht nur die unmittelbar interessierten Unternehmen auf eine ver- stärkte wirtschaftliche Zusammenarbeit mit China. Vor dem Hintergrund der beschriebenen Reputationsrisiken bemühen sich inzwischen zahlreiche europäische, japanische und US-amerikanische Unternehmen darum, die Einhaltung grundlegender Sozialstandards auch in China sicherzustellen. Um die Glaubwürdigkeit ihrer Aktivitäten zu untermauern, werden runde Tische eingerichtet, unterschiedliche Formen des Dialogs initiiert und Auditierungen und Zertifizierungen bei einer noch kleinen, aber doch zunehmenden Zahl von chinesischen Zulieferfirmen durchgeführt.

Für einzelne Manager in chinesischen Betrieben muss es in Teilen verwirrend und aus ihrer Sicht womöglich sogar ehrverletzend sein, sich nach unterschiedlichsten privaten Sozialstandards teilweise auch mehrfach zertifizieren zu lassen, damit die weltweit agierenden Markenartikelunternehmen ihre Produkte in Europa mit gutem Gewissen verkaufen können. Dies dürfte ein wesentlicher Grund dafür gewesen sein, dass die oberste staatliche Zertifizierungsaufsicht in China Ende 2004 angekündigt hat, die Entwicklung eines eigenen Standards in Angriff zu nehmen.

\section{Neues CSR-Verständnis}

Inzwischen hat es den Anschein, als ob Teile der politischen Elite in China erkannt haben, dass zu einer weichen Landung des rasanten Wirtschaftswachstums auch das weiche Steuerungskonzept einer Corporate Social Responsibility beitragen kann. Es scheint sich die Auffassung durchzusetzen, dass CSR im globalen Wettbewerb um die Gunst von Investoren und Auftragnehmern, aber auch bei den komplizierter werdenden Verhandlungen in der Welthandelsorganisation künftig eine wichtige strategische Bedeutung spielen kann. Die systematische Beobachtung wichtiger chinesischer Online-Medien zeigt jedenfalls, dass insbesondere 2005 und 2006 eine deutliche Intensivierung der Diskussionen über CSR in China stattgefunden hat (Nordmann 2006).

Bereits 2003 hatte die chinesische Regierung damit begonnen, im Umweltschutz vorbildlich agierende staatliche 
Unternehmen zu identifizieren, die zugleich profitabel wirtschaften. Diese CSRVorreiter aus verschiedensten Branchen werden als gute Beispiele für andere chinesische Betriebe dargestellt. Nach oft wiederholter Aussage des chinesischen staatlichen Hauptamtes für Umweltschutz ist betrieblicher Umweltschutz ein wichtiger Schritt auf dem Weg zu einer umweltfreundlichen und ressourcenschonenden Gesellschaft.

Im September 2005 fand in Beijing das erste europäisch-chinesische CSR-Forum statt. In diesem Rahmen erklärten hohe Staatsbeamte, dass man CSR sowohl den Unternehmen, als auch dem Staatsapparat nahebringen wolle (ORSE 2006).

Auch der Global Compact wird vonseiten der chinesischen Regierung ausdrücklich beworben. Bislang haben sich 70 chinesische Unternehmen zu den Prinzipien des Compacts bekannt. Im August 2006 wurde zudem die Chinese Association for Corporate Social Responsibility gegründet, der bislang etwa 20 Unternehmen aus dem In- und Ausland angehören.

Schließlich beteiligt sich China konstruktiv an den Bemühungen der International Organization for Standardization, eine international gültige CSR-Guideline zu entwickeln.

Eine erste Untersuchung zum CSRVerständnis und zur CSR-Praxis von 890 chinesischen Unternehmen verdeutlichte, dass der Begriff CSR vielen der hier befragten großen chinesischen Unternehmen durchaus bekannt ist. Die Auffassungen von CSR sind allerdings diffus und enthielten nahezu alle Vorschläge, die von verstärkten gesetzlichen Vorschriften bis zu Wohltätigkeitsinitiativen reichen (China Daily 2006). Dass es schon heute Unternehmen in China gibt, die an einem dezidierten CSR-Konzept arbeiten, zeigen die ersten beiden Nachhaltigkeitsberichte aus China. Sie stammen vom Energieversorger State Power Corporation und der Shanghai Pudong Development Bank. Nach chinesischen Medienberichten sollen auch zahlreiche weitere Unternehmen erste Ansätze zur CSR-Berichterstattung aufweisen.
Als besonders bemerkenswert kann die aktuelle Aufnahme eines chinesischen Unternehmens, der Bio-Treat Technology, in den sogenannten Natur-Aktienindex gelten. Der Natur-Aktienindex gilt innerhalb des nachhaltigen Investments weithin als Maßstab für grüne Geldanlagen und zeichnet sich durch konsequente ökologisch-soziale Auswahlkriterien aus und setzt mit diesem Schritt sicher ein Signal, das über den Einzelfall hinausgeht.

\section{Explizite und implizite CSR}

Auch wenn chinesische Politiker, Wirtschaftsexperten und einige Wissenschaftler den Begriff der Corporate Social Responsibility verwenden, muss beachtet werden, dass hier in der Regel eine sehr eigenständige Interpretation zugrunde liegt (He 2006). CSR wird in China als Baustein auf dem Weg zu einer harmonischen Gesellschaft gesehen, die angesichts der krassen sozialen Unterschiede der chinesischen Übergangsgesellschaft und des ökologischen Raubbaus als integrierende Wirtschafts- und Sozialvision dient. Anders als in der US-amerikanisch dominierten und auch in Europa vorherrschenden Interpretation von CSR wird in China auch die Einhaltung von einschlägigen gesetzlichen Vorschriften als CSR-Politik verstanden. Angesichts der in China vielfach anzutreffenden "bad governance" kommt den Anstrengungen der chinesischen Regierung insgesamt eine große Bedeutung zu, Unternehmen „zu motivieren“, sich an die vorhandenen nationalen gesetzlichen Vorschriften, aber auch an internationale Rechtsvorschriften zu halten.

Es scheint, dass die von Matten/Moon (2005) in die Diskussion gebrachte Unterscheidung von expliziter (freiwilliger) und impliziter (gesetzlich geregelter) CSR, die auf der Grundlage gesetzlicher Regelungen soziale und ökologische Zielsetzungen umsetzt, zum Verständnis der sich abzeichnenden chinesischen CSR-Initiativen besonders wichtig ist. Denn das Verhältnis von impliziter und expliziter CSR beschreibt für China ein deutliches Konfliktpotenzial. So beobachtet die Internationale Gewerkschaftsunion IUL kritisch, dass führende Global Player gegen den Entwurf eines neuen Arbeitsgesetzes in China Sturm laufen, um lieber flexibel die eigenen Codes of Conduct anzuwenden (IUL 2006). Insgesamt kann davon ausgegangen werden, dass China in den nächsten Jahren vermehrt eigenständige Beiträge zur Entwicklung eines tragfähigen globalen CSR-Verständnisses in die internationale Diskussion einbringen wird.

\section{Literatur}

China Daily: Survey reveals CSR not fully understood. Internet:

http://english.peopledaily.com.cn/200610/27/ print20061027_315634.html. 2006.

$\mathrm{He}$, Zyhiyi: Corporate Social Responsibility - Herausforderungen und Ansporn für die chinesische Wirtschaft. In: Schoenheit, I. / Iwand, W. / Kopp, R. (Hrsg): Corporate Social Responsibility - Verantwortung für nachhaltiges Wirtschaften in China. Berlin 2006. S. 24-3.

IUL: China und die globale Lobby der Ausbeuterbetriebe. Internet: http://www.iuf.org/cgibin/editorials/db.cgi?db=default\&ww=1\&uid= default\& $\mid D=508 \&$ view_records $=1 \&$ de $=1.2006$.

Matten, D. / Moon; J.: A Conceptual Framework for Understanding CSR. In: Habisch, A. et. al (Hrsg.): Corporate Social Responsibility Across Europe. Berlin 2005. S. 335-357.

Nordmann, A.: Monitoring von CSR-relevanten Entwicklungen in China. In: Schoenheit, I. / Iwand, W. / Kopp, R. (Hrsg): Corporate Social Responsibility - Verantwortung für nachhaltiges Wirtschaften in China. Berlin 2006. S. 95 107.

ORSE: Corporate Social Responsibility in China. Study 9. Paris 2006.

Schoenheit, I.: Corporate Social Responsibility Deutsche und internationale Perspektiven am Beispiel China. In: Schoenheit, I. / Iwand, W. / Kopp, R. (Hrsg): Corporate Social Responsibility - Verantwortung für nachhaltiges Wirtschaften in China. Berlin 2006. S. 9-24.

Schoenheit, I.: Politischer Konsum - ein Beitrag zum faustischen Konsumverhalten. In: Jäckel, M. (Hrsg.): Ambivalenzen des Konsums und der werblichen Kommunikation. Wiesbaden 2007 (im Druck).

\section{AUTOR + KONTAKT}

Dr. Ingo Schoenheit ist geschäftsführender Vorstand des Instituts für Markt-UmweltGesellschaft (imug) an der Universität Hannover und Axel Nordmann ist Projektleiter ,Internationales Issue Monitoring' der imug Beratungsgesellschaft mbH.

IMUG, Brühlstraße 11, 30169 Hannover. Tel.: 0511/91115-0, Fax: 0511/91115-9, E-Mail: schoenheit@imug.de, nordmann@imug.de, Website: www.imug.de 
(c) 20I0 Authors; licensee IÖW and oekom verlag. This is an article distributed under the terms of the Creative Commons Attribution Non-Commercial No Derivates License (http://creativecommons.org/licenses/by-nc-nd/3.o/), which permits unrestricted use, distribution, and reproduction in any medium, provided the original work is properly cited. 